Abstract \#2: Poster (Research Study)

\title{
Evidence from Consultation Defines the Difference Between Clinical Doctorates and the Ph.D. in Clinical Laboratory Science
}

Since the inception of the doctorate in clinical laboratory science (DCLS), universities sponsoring this new profession have struggled with its academic placement. The $\mathrm{PhD}$ is considered the highest achievement level and oriented toward research. Clinical/practice doctorates are considered applied though still at the doctoral level and classified as terminal degrees for many healthcare professions. Rutgers University, the first to offer the DCLS, opted to launch this profession as a clinical doctorate, conceiving the practice to be applied and patient/consumer focused. During completion of the first DCLS curriculum, evidence emerged describing an additional curriculum need to augment competencies in clinical research equivalent to competencies associated with the Ph.D. During the DCLS residency year, the Consultation Model was implemented in four clinical settings: Consumer Information Response (CIR) a national consultation network, Diagnostic Management Team (DMT) laboratory consultation, Patient Care Rounding Team (PCRT) clinical service consultation, and Utilization Review (UR) EHR and LIS review consultation. Data were collected on multiple variables: provider type, diagnosis, diagnostic question, testing cycle phase, treatment phase, and complexity (hand-offs and/or logic steps). Analyses indicated the need for additional clinical research competencies, particularly data analytics associated with data warehouses built from HER/LIS elements, needed to develop diagnostic algorithms supporting treatment paths. Curriculum developing adding these research competencies to the DCLS curriculum through research projects is underway; progression to completion will result in the award of the DCLS/Ph.D. Doctoral students will be accepted into either program but must demonstrate aptitude in clinical research to progress to the Ph.D. 